\title{
Análise epidemiológica comparativa entre transplante hepático de doadores vivos e doadores mortos nos últimos 5 anos no Rio de Janeiro.
}

\section{Comparative epidemiological analysis between liver transplantation from living and dead donors in the last 5 years in Rio de Janeiro.}

Maria Carolina da Silva Gaspar'; Júlia da Silveira Pacheco Ferraz¹; Mariana do EspíritoSanto e Santos¹; Thayssa Vasconcellos Guide ${ }^{1}$; Cristina Maria Monteiro Dantas ${ }^{2}$

Como citar esse artigo. Gaspar, M.C.S

Ferraz, J.S.P; Santos, M.E; Guide, T.V;

Dantas, C.M.M. Análise epidemiológica comparativa entre transplante hepático de doadores vivos e doadores mortos nos últimos 5 anos no Rio de Janeiro. Revista de Saúde. 2021 Abr.Jul.; 12 (2): 33-36.

\section{Resumo}

O transplante de fígado é o tratamento de escolha para pacientes com insuficiência hepática, quando não há mais proposta terapêutica conservadora efetiva. Os objetivos do transplante são melhorar a qualidade de vida do paciente e prolongar sua sobrevida. Este órgão pode ser doado a partir de um cidadão vivo ou falecido. O propósito deste estudo é realizar uma análise comparativa, no Estado do Rio de Janeiro, entre cada procedimento, considerando dados como número total de cirurgias, custo médio, taxa de mortalidade e tempo médio de internação. Segundo os resultados, no Rio de Janeiro, $91,18 \%$ dos transplantes utilizaram órgão de doadores falecidos e 8,82\% de vivos.Os custos foram de R\$ 82.162,94 para procedimento intervivos e R $\$ 94.317,72$ para falecidos. A média de internação foi de 13,9 dias para o procedimento entre vivos e 10,5 dias para doador morto. A taxa de mortalidade e óbitos foram $8,97 \%$ e 7 , respectivamente, para doadores vivos e 10,55\% e 85 para doadores mortos. Apesar da predominância de doadores falecidos, esse procedimento apresenta empecilhos como a resistência das famílias a doação e problemas organizacionais do órgão responsável.E, ainda, existe um temor por parte dos candidatos a doadores em vida com sua saúde e complicações cirúrgicas. Sendo assim, há necessidade de conscientizar a população sobre a importância de ser doador, esclarecer as famílias o real significado da morte encefálica e sanar possíveis dúvidas. Somente o estímulo a ambos os tipos de doação poderá reduzir as filas de espera e salvar vidas.

Palavras-chave: Transplante de fígado; Transplante de doador vivo; Transplante de doador morto; Fígado de doador vivo e ética.

\begin{abstract}
Liver transplantation is the treatment of choice for patients with liver failure, when there is no more effective conservative therapeutic proposal. The goals of transplantation are to improve the patient's quality of life and prolong his survival. This organ can be donated from a living or deceased citizen. The purpose of this study is to perform a comparative analysis, in the State of Rio de Janeiro, between each procedure, considering data such as total number of surgeries, average cost, mortality rate and average length of stay. According to the results, in Rio de Janeiro, $91.18 \%$ of transplants used organs from deceased donors and $8.82 \%$ from living ones. The costs were R \$ 82,162.94 for interventional procedures and R \$ 94,317.72 for deceased. The average hospital stay was 13.9 days for the live procedure and 10.5 days for the dead donor. The mortality and death rates were $8.97 \%$ and 7 , respectively, for living donors and $10.55 \%$ and 85 for dead donors. Despite the predominance of deceased donors, this procedure presents obstacles such as families' resistance to donation and organizational problems of the responsible agency. And yet, there is a fear on the part of prospective donors living with their health and surgical complications. Therefore, there is a need to make the population aware of the importance of being a donor, to clarify to families the real meaning of brain death and to resolve possible doubts. Only the encouragement of both types of donation can reduce waiting lines and save lives.
\end{abstract}

Keywords: Liver transplantation; Living donor transplantation; Dead donor transplantation; Living donor liver and ethic.

\section{Introdução}

De acordo com o Ministério da Saúde, a definição de transplante é "um procedimento cirúrgico que consiste na reposição de um órgão ou tecido de uma pessoa doente (receptor) por outro órgão ou tecido são de um doador, vivo ou morto"1. Sabe-se que o Brasil, hoje, é uma das referências mundiais em transplante, ficando atrás apenas dos Estados Unidos da América ${ }^{1}$. O Brasil possui 555 estabelecimentos de saúde e
1.376 equipes médicas autorizados pela Coordenação Geral do Sistema Nacional de Transplantes a realizar tal procedimento ${ }^{2}$. Cerca de $96 \%$ dos transplantes são financiados inteiramente pelo Sistema Único de Saúde (SUS) ${ }^{1}$.

Um dos órgãos passíveis de ser transplantado é o fígado ${ }^{1}$. A história dessa cirurgia começa em março de 1963, quando foi realizada pela primeira vez no mundo por Starzl et al, em um menino de três anos com atresia biliar, que não sobreviveu. ${ }^{3}$ No Brasil, essa terapia foi 
inaugurada em 1968 por Machado no Hospital das Clínicas da Faculdade de Medicina da Universidade de São Paulo, também não obtendo sucesso. ${ }^{3}$ De acordo com a Associação Brasileira de Transplante de órgãos (ABTO), no último ano foram realizados 24.131 transplantes no Brasil, sendo 2.245 hepáticos ${ }^{4}$.

O transplante hepático no Brasil encontra-se em expansão ${ }^{5}$. Esse fato pode ser explicado pelo aumento da população brasileira ${ }^{5}$ e pelo forte empenho do governo federal em divulgar informações sobre doação de órgãos, conscientizando a população sobre sua importância ${ }^{2,3,5}$. O país alcançou um número histórico de 13,2 doadores por milhão de habitantes ${ }^{3}$. Todavia, o programa de transplante brasileiro necessita ser continuamente aperfeiçoado, principalmente no quesito organização, para que um número cada vez maior de pacientes seja contemplado ${ }^{2}$.

O transplante hepático, assim como outros órgãos, pode ser realizado a partir de um órgão provindo de pessoa falecida ou pessoa viva, uma vez que doar parte dele não acarreta danos ao doador ${ }^{1}$. Esse procedimento é também considerado um excelente método para tratamento de insuficiência hepática terminal, em termos de simplicidade, economia e sucesso ${ }^{6}$. Sendo assim, pacientes cirróticos ou com carcinoma hepatocelular dentro dos critérios de Milão, são alguns exemplos dos que podem ser beneficiados por esse método. ${ }^{3}$ Além disso, a hepatite $\mathrm{C}$ é em todo mundo, umas das principais indicações para tal procedimento ${ }^{5}$. As indicações para o transplante de fígado podem ser divididas em 4 categorias principais: insuficiência hepática aguda, doença hepática crônica, síndromes variantes e câncer primário de fígado ${ }^{7}$. Os pacientes precisam preencher critérios rigorosos para ser alocado na fila de espera ${ }^{7}$ e esse processo deve ser extremamente cuidadoso ${ }^{8}$. No Brasil, a alocação de pacientes segue o critério de gravidade da doença, baseando-se na pontuação MELD (Model for End-StageLiverDisease) ${ }^{8}$.

Dados da ABTO, que mostram uma necessidade de transplante hepático equivalente a 5.212 casos em 2019, enquanto apenas 2.245 pacientes foram contemplados no mesmo ano ${ }^{4}$. Há grande resistência das famílias em consentir a doação de órgãos de entes falecidos ${ }^{1}$. Com isso, os doadores vivos foram e são de suma importância para complementar a carência de órgãos ${ }^{6}$. Entretanto, os órgãos provenientes da doação por indivíduos com morte encefálica ainda representam a maior parte do substrato para o transplante ${ }^{9}$, como visto em 2019 no Brasil, onde do total de transplantes hepáticos aproximadamente $93 \%$ foi feito a partir desses doadores ${ }^{4}$. Sendo assim, esse trabalho objetiva uma análise epidemiológica entre os dois tipos de doadores em transplantes hepáticos, no Estado do Rio de Janeiro, nos últimos 5 anos, considerando dados como número total de cirurgias, custo médio, taxa de mortalidade e tempo médio de internação.

\section{Material e Métodos}

Foram analisados dados da plataforma DATASUS, Sistema de Informações Hospitalares do SUS (SIH/SIS) do Ministério da Saúde, dentro do período compreendido de maio de 2015 a abril de 2020. Desse site, coletou-se informações como quantidade de transplantes, média de dias de internação, óbitos, taxa de mortalidade e custos totais por procedimento (Tabela 1). Foi realizada uma revisão bibliográfica entre o período de 2005 a 2020 através de busca no banco de dados nos sites PubMed, Scielo e Google Acadêmico, utilizando as palavras-chave: Transplante de fígado; Transplante de doador vivo; Transplante de doador morto; Fígado de doador vivo e ética. Os idiomas selecionados foram: português e inglês.

Tabela 1. Método da pesquisa no site DATASUS.

\begin{tabular}{|c|c|}
\hline Passo 1 & $\begin{array}{l}\text {-Acessar DATASUS (http://www2.datasus.gov.br) } \\
\text {-Informações de Saúde }\end{array}$ \\
\hline Passo 2 & $\begin{array}{l}\text {-Acessar Assistência à saúde } \\
\text { •Produção Hospitalar (SIH/SUS) }\end{array}$ \\
\hline Passo 3 & $\begin{array}{l}\text {-Dados Consolidados AIH (RD), por local de internação, a } \\
\text { partir de } 2008 \\
\text {-Abrangência Geográfica: Selecionar Brasil por Região e } \\
\text { Unidade da Federação }\end{array}$ \\
\hline Passo 4 & $\begin{array}{l}\text { - Linha: Unidade da Federação } \\
\text { •Coluna: Não ativa } \\
\text { •Conteúdo: Variáveis desejadas (internações, valor médio } \\
\text { internações, média permanência, óbitos, taxa de } \\
\text { mortalidade) }\end{array}$ \\
\hline Passo 5 & $\begin{array}{l}\text { •Período: maio de } 2015 \text { até abril de } 2020 \text { (selecionar cada } \\
\text { mês) }\end{array}$ \\
\hline Passo 6 & $\begin{array}{l}\text {-Unidade de Federação: Rio de Janeiro } \\
\text {-Procedimento: código } 0505020050 \text { - transplante hepático } \\
\text { órgão de doador falecido e código } 0505020068 \text { - } \\
\text { transplante hepático órgão de doador vivo (fazer de cada } \\
\text { procedimento separadamente) }\end{array}$ \\
\hline
\end{tabular}

\section{Resultados}

De acordo com os dados do DATASUS ${ }^{10}$, foram realizados nos últimos 5 anos no Rio de Janeiro um total de 884 transplantes hepáticos, sendo 78 de doadores vivos $(8,82 \%)$ e $806(91,18 \%)$ de doadores mortos. Os custos por internação para realizar tais procedimentos foram $\mathrm{R} \$ 82.162,94 \mathrm{com}$ doadores vivos e R\$ 94.317,72 com doadores mortos, totalizando $\mathrm{R} \$ 176.480,66$. O tempo médio de internação, foi de 13,9 dias para doadores em vida e 10,5 dias para doadores cadáveres. O número de óbitos foi de 7 pacientes para doadores vivos, com uma taxa de mortalidade de $8,97 \%$ e de 85 pacientes para doadores falecidos, sendo a taxa de mortalidade igual a 10,55\%(Tabela 2). 
Tabela 2. Total de procedimentos, custos por internação, tempo médio de internação, taxa de mortalidade e óbitos por unidade da federação (Rio de Janeiro).

Procedimento 0505020050 transplante hepático (órgão de doador falecido); Procedimento 0505020068 transplante hepático (órgão de doador vivo).

Período: Maio/2015 - Abril/2020.

\begin{tabular}{lcc}
\hline & Doador Falecido & Doador Vivo \\
\hline Total de Procedimentos & 806 & 78 \\
\hline Custos por Internação (R\$) & $94.317,72$ & $82.162,94$ \\
\hline Tempo Médio de Internação & 10,5 & 13,9 \\
\hline Taxa de Mortalidade & 10,55 & 8,97 \\
\hline Óbitos & 85 & 7
\end{tabular}

Fonte: Ministério da Saúde - Sistema de Informações Hospitalares do SUS (SIH-SUS) ${ }^{10}$

\section{Discussão}

A maioria dos transplantes hepáticos realizados pelo SUS, no Rio de Janeiro, assim como no mundo?, são feitos a partir de órgãos provindos de doadores mortos. Entretanto, esse tipo de doação apresentou custos mais elevados, apesar de possuir um tempo médio de internação menor, se comparado com o procedimento provindo de doadores vivos, o que diverge de outros estudos ${ }^{11}$. A análise dos custos deve levar em consideração variáveis relacionadas a gravidade da doença de cada paciente ${ }^{8}$, bem como os orçamentos e as políticas de compras de insumos de cada hospital ${ }^{12}$.

O Sistema Nacional de Transplante (SNT) do Brasil passa por problemas organizacionais $\mathrm{Como}^{13}$ : falta de acesso a nível nacional de exames para diagnosticar morte encefálica; impasses na manutenção e transporte de órgãos; problemas na gestão das Centrais de Notificação e Captação e Distribuição de Órgãos(CNCDO's) ${ }^{2}$. Todos esses problemas contribuem para que menos de $50 \%$ dos casos sejam notificados a central responsável e somente $12,2 \%$ acabem se tornando efetivamente doadores ${ }^{2}$. A consequência direta é uma longa fila de espera, contribuindo para o agravamento das condições clínicas dos pacientes que necessitam do transplante e aumento nas taxas de mortalidade ${ }^{14}$. O Rio de Janeiro, apresenta a segunda pior posição na lista de espera para transplante hepático no Brasil ${ }^{13}$.Analisando-se os dados observa-se que o transplante intervivos, embora pouco realizado no Rio de Janeiro ( $8,82 \%$ dos casos) seria uma alternativa para minimizar a longa espera por esse órgão ${ }^{15}$.

Alguns estudos encontraram índices de complicações gerais mais altos $(26 \%)$ e mais graves (2\%), inclusive com necessidade de novo procedimento, no transplante intervivos ${ }^{16,17}$. Entretanto, no Estado do Rio de Janeiro, a taxa de mortalidade foi maior nos procedimentos com órgão de doador falecido, possivelmente justificada pela maior utilização desse tipo de doadores.

Existe ainda uma dificuldade de compreensão por parte das famílias do significado da morte cerebral. Muitas não autorizam a doação por acreditarem que enquanto existe "batimento cardíaco" há vida1. Por outro lado, existe um temor por parte dos candidatos a doadores em vida, de que haja um prejuízo a longo prazo para sua saúde, ou que ocorram complicações no procedimento cirúrgico ao qual deverão ser submetidos ${ }^{18}$. As complicações mais frequentes para o doador vivo são respiratórias, eventos tromboembólicos e sangramentos pós-operatórios $(4,3 \%)^{15}$, com umataxa demortalidade variando de 0,1 a $0,3 \%{ }^{15}$. Além disso, os critérios de seleção dos possíveis doadores são muito rigorosos (não apresentar nenhuma comorbidade, possuir entre 18 e 60 anos, ter compatibilidade sanguínea, assinar um termo de consentimento e se não for membro da família até $4^{\circ}$ grau necessita de uma autorização judicial, entre outros $)^{1,19,20}$ e se tornaram ainda mais rigorosos com a pandemia do Coronavírus ${ }^{21}$. Todos os fatos expostos acima contribuem para limitar o número de doadores.

\section{Conclusão}

O transplante de fígado possibilita a cura para pacientes com insuficiência hepática, sem resposta a terapêutica conservadora. Mundialmente, assim como no Rio de Janeiro, utiliza-se predominantemente para tal procedimento órgãos oriundos de doadores mortos. Medidas organizacionais, de conscientização e esclarecimento aos possíveis doadores e famílias, são necessárias para estimular o transplante intervivos, o que ajudaria a diminuira defasagem entre a disponibilidade de órgãos e número de pacientes na fila de espera.

\section{Referências}

1. Ministério da Saúde. Saúde de A a Z. Doação de Órgãos: transplantes, lista de espera e como ser doador [Internet]. Brasil; 2020 [Citado em: 22 jun 2020]. Disponível em: https://www.saude.gov.br/saude-de-a-z/doacao-deorgaos

2

Garcia CD, Pereira JD, Garcia VD, organizadores. Doação e transplante de órgãos e tecidos. 1. ed. São Paulo: Segmento Farma; 2015. 560 p.

3. Meirelles Júnior RF, Salvalaggio P, Rezende MB, et al. Liver transplantation: history, outcomes and perspectives. Einstein 2015;13(1):149152 .

4. Associação Brasileira de Transplantes de Órgãos. Dimensionamento dos Transplantes no Brasil e em cada estado: Registro Brasileiro de Transplantes [Internet]. 2019; [Citado em: 31 jul 2020]. Disponível em: https://site.abto.org.br/wp-content/uploads/2020/06/RBT-2019-leitura-1.pdf

5. Trotter JF. Liver transplantation around the world. CurrOpinOrganTransplant 2017;22(2):123-127.

6. Bozkurt B, Dayangac M, Tokat Y. Living Donor Liver Transplantation Chirurgia2017;112(3):217-228. 
7. Halliday N, Westbrook RH. Livertransplantation: need, indications, patientselectionandpre-transplantcare. Br J Hosp Med 2017;78(5):252-259.

8. Rodríguez S, Motta F, Balbinoto Neto G, Brandão A. Evaluationandselectionof candidates for livertransplantation: aneconomic perspective. ArqGastroenterol2020;57(1):31-38.

9. Jay CL, Skaro AI, Ladner DP, et al. Comparative effectiveness of donation after cardiac death versus donation after brain death liver transplantation: Recognizing who can benefit. LiverTranspl 2012;18(6):630640.

10. Ministério da Saúde. Departamento de Informática do SUS (DATASUS). Informações de Saúde (Tabnet) - Procedimentos Hospitalares do SUS [Internet]. 2020 [Citado em: 20 jul. 2020]. Disponível em: http:// tabnet.datasus.gov.br/cgi/deftohtm.exe?sih/cnv/qiuf.def

11. Coelho JCU, TrubianPS, Freitas ACT,Parolin MB, Schulz GJ, Martins EL. Comparação entre o custo do transplante hepático cadavérico e o intervivos. RevAssoc Med Bras 2005;51(3):158-163.

12. Souza AB, Rodriguez S, Motta FLD, Brandão ABM, Marroni CA. The costofadultlivertransplantation in a referral center in southernBrazil. ArqGastroenterol. 2019;56(2):165-171.

13. Marinho A, Cardoso Sde S, Almeida VV.Geographicdisparities in organtransplantation in Brazil. CadSaude Publica2010;26(4):786-796.

14. Lauterio A, Di Sandro S, Giacomoni A, De Carlis L. The role of adult living donor liver transplantation and recent advances. Expert Rev Gastroenterol Hepatol2015;9(4):431-445.

15. Gorgen A, Goldaracena N, Zhang W, et al. Surgical Complications after Right Hepatectomy for Live Liver Donation: Largest Single-Center Western World Experience. Semin Liver Dis 2018;38(2):134-144.

16. Goldaracena N, Jung J, Aravinthan AD, et al. Donor outcomes in anonymous live liver donation. J Hepatol 2019;71(5):951-959.

17. Fisher RA. Living donor liver transplantation: eliminating the wait for death in end-stage liver disease? Nat Rev Gastroenterol Hepatol2017;14(6):373-382.

18. Baker TB. Living liver donation, donor safety, and social media: Preparing for a new frontier. LiverTranspl2017;23(2):131-132.

19. Lieber SR, Schiano TD, Rhodes R. Should living donor liver transplantation be an option when deceased donation is not? J Hepatol 2018;68(5):1076-1082.

20. Goldaracena N, Barbas AS. Living donorlivertransplantation. CurrO pinOrganTransplant2019;24(2):131-137.

21. Lau G, Ward JW. SynthesisofLiverAssociationsRecommendations for HepatologyandLiverTransplantCareDuringthe COVID-19 Pandemic. ClinLiverDisease2020;15(5):204-209. 